\title{
On Solutions of Fractional Order Boundary Value Problems with Integral Boundary Conditions in Banach Spaces
}

\author{
Hussein A. H. Salem ${ }^{1,2}$ and Mieczysław Cichon ${ }^{3}$ \\ ${ }^{1}$ Department of Mathematics, Faculty of Sciences, Alexandria University, Alexandria, Egypt \\ ${ }^{2}$ Faculty of Sciences, Taibah University, Yanbu, Saudi Arabia \\ ${ }^{3}$ Faculty of Mathematics and Computer Science, Adam Mickiewcz University, Umultowska 87, 61-614 Poznan, Poland
}

Correspondence should be addressed to Hussein A. H. Salem; hssdina@alex-sci.edu.eg

Received 28 August 2012; Accepted 6 April 2013

Academic Editor: Gennaro Infante

Copyright (C) 2013 H. A. H. Salem and M. Cichoń. This is an open access article distributed under the Creative Commons Attribution License, which permits unrestricted use, distribution, and reproduction in any medium, provided the original work is properly cited.

\begin{abstract}
The object of this paper is to investigate the existence of a class of solutions for some boundary value problems of fractional order with integral boundary conditions. The considered problems are very interesting and important from an application point of view. They include two, three, multipoint, and nonlocal boundary value problems as special cases. We stress on single and multivalued problems for which the nonlinear term is assumed only to be Pettis integrable and depends on the fractional derivative of an unknown function. Some investigations on fractional Pettis integrability for functions and multifunctions are also presented. An example illustrating the main result is given.
\end{abstract}

\section{Introduction}

The theory of boundary value problems is one of the most important and useful branches of mathematical analysis. Boundary value problems of various types create a significant subject of several mathematical investigations and appear often in many applications, especially in solving numerous problems in physics and engineering. For example, heat conduction, chemical engineering, underground water flow, thermoelasticity, and plasma physics can be reduced to nonlocal problems with integral boundary conditions. For boundary value problems with integral boundary conditions and comments on their importance, we refer the reader to [1$3]$ and the references therein.

The class of boundary value problems with integral boundary conditions considered below contains as special cases numerous two, three, multipoint, and nonlocal boundary value problems. Such problems are mainly investigated when considering functions satisfying some conditions expressed in terms of the strong topology of a Banach space $E$. We will investigate the case, when functions are not strongly continuous and strongly integrable. In this situation we need to introduce more general notion of a solution. We should note that the considered case seems to be a natural case and cover many particular cases considered for both the strong and weak topologies (cf. Lemma 19). A more general notion of solutions allows us to solve the problem under very general assumptions, not so restrictive as before (see our last section).

In contrast to the classical approach for the theory of boundary value problems, the theory for fractional order BVP's is still developing one and not satisfactorily described. It is caused by the fact that it is very difficult to find convenient and handy conditions ensuring the existence of solutions of several nonlinear boundary value problems of fractional order. In the considered case of a weak topology on $E$ our results form a relatively new branch of investigations.

As a pursuit of this, some sufficient conditions for the existence of solutions are presented for the following nonlinear $m$-point boundary value problem of fractional type:

$$
\begin{gathered}
D^{\alpha} u(t)+f\left(t, u(t), D^{\beta} u(t)\right)=0, \\
t \in[0,1], \quad \alpha \in(1,2], \quad \beta \in(0,1), \quad \alpha>1+\beta, \\
u(1)+\int_{0}^{1} \mathfrak{I}(\tau) u(\tau) d \tau=l, \quad u(0)=0,
\end{gathered}
$$


where $x$ takes values in a Banach space $E$ and $l \in E$. Here $\mathfrak{I} \in L^{q}[0,1]$ for some $q \in[1, \infty]$ and $D^{\alpha}$ denotes the pseudo fractional differential operator of order $\alpha$ (to be described later). We will assume that $f$ is a vector-valued Pettis integrable function on $[0,1]$. We remark the following:

(1) for real-valued functions with $\alpha, \beta \in \mathbb{N}:=\{1,2,3$, $4, \ldots\}$, we have problems studied in, for example, $[4$, 5],

(2) for real-valued functions with $\mathfrak{\Im} \equiv 0$ and $\alpha, \beta \in \mathbb{N}$, we have problems studied in, for example, [6-10]; see also the references therein,

(3) for real-valued functions $\mathfrak{\Im} \equiv 0$ and when the function $f$ is independent of the fractional derivatives, then we have problems studied in, for example, $[1,11-$ 13],

(4) in abstract spaces (for vector-valued functions) with $\mathfrak{I} \equiv 0$ and $\alpha, \beta \in \mathbb{N}$, we have problems studied in, for example, [14-19],

(5) in abstract spaces with conditions related to the weak topology on $E$ and when the vector-valued function $f$ is independent of the fractional derivatives, then we have a problems studied in, for example, $[20,21]$.

In comparison with the existence results in the above list, our assumptions seem to be more natural. In contrast to earlier results, we drop the requirement that $f$ is a real-valued function independent of the fractional derivatives and we consider the case of vector-valued Pettis, but not necessarily Bochner, integrable functions. As we mentioned above, the assumptions in the existence theorem are expressed in terms of the weak topology. Such a result does not appear in the earlier literature and so it seems to be new. We collect all interesting properties for the fractional Pettis integral. Moreover, we are able also to start some studies for multivalued fractional boundary value problems with Pettis-integral boundary conditions and fractionally Pettis integrable multifunctions.

In the paper we stress also on comparison results for Pettis integrals and fractional Pettis integrals. This is also done for the multivalued integrals and seems to be interesting by itself, independently of applicability of our results. The properties of fractional integral operators on the spaces of Pettis integrable functions as well as on some of its subspaces are also investigated.

Finally, we remark that, in the Banach spaces, the existence of solutions of some boundary value problems of fractional orders has been considered in terms of Pettis integrals, for the first time, by Salem [20]. In this paper, for clarity of proofs, we restrict ourselves to the case of reflexive spaces, but it is easy to extend our results for nonreflexive spaces by putting contraction hypothesis with respect to some measure of weak noncompactness and by using appropriate fixed point theorem (cf. [22]). Nevertheless, all auxiliary results in this paper are not restricted to reflexive spaces.

The question of proving the existence of solutions to the problem (1) reduces to proving the existence of solutions of a Fredholm integral equation. Since the space of all Pettis integrable functions is not complete (in general), we restrict our attention to the case of weakly continuous solution of the Fredholm integral equation (modeled off the problem (1)); hence we are ready to find the so-called pseudo-solutions of the problem (1) (cf. [22, 23]).

\section{Preliminaries and Auxiliary Results}

For the sake of the reader's convenience here we collect a few facts which will be needed further on. Let $I=[0,1]$. According to the custom $L^{p}(I), 1 \leq p \leq \infty$ will denote the Banach space of real-valued measurable functions $x$ defined on $I$. Let $L^{\infty}(I)$ denote the Banach space of real-valued essentially bounded and measurable functions defined on $I$. Through the paper, $E$ is considered to be a Banach space with norm $\|\cdot\|$ and with its dual space $E^{*}$. Moreover, let $E_{w}=(E, w)=\left(E, \sigma\left(E, E^{*}\right)\right)$ denote the space $E$ with its weak topology. By $C[I, E]$ we will denote the Banach space of strongly continuous functions $x: I \rightarrow E$ endowed with a standard $\|x\|_{0}=\sup _{t \in I}\|x(t)\|$, while $P[I, E]$ denotes the space of all $E$-valued Pettis integrable functions in the interval $I$ (see $[24,25]$ for the definition). Let us also recall that a function $h: E \rightarrow E$ is said to be weakly-weakly sequentially continuous if $h$ takes each weakly convergent sequence in $E$ into weakly convergent sequence in $E$. We point out that a bounded weakly measurable function $x: I \rightarrow E$ need not to be Pettis integrable even if $E$ is reflexive. However, in reflexive Banach spaces, the weakly measurable function $x: I \rightarrow E$ is Pettis integrable if and only if $\varphi(x(\cdot))$ is Lebesgue integrable on $I$ for every $\varphi \in E^{*}[26]$.

Let us recall some basic facts. The following Mazur's lemma can be found in $[24,26]$.

Lemma 1. A convex subset of a normed space $E$ is closed if and only if it is weakly closed.

A simple consequence of the Hahn-Banach theorem is as follows.

Proposition 2. Let $E$ be a normed space with $x_{0} \neq 0$. Then there exits $\varphi \in E^{*}$ with $\|\varphi\|=1$ and $\varphi x_{0}=\left\|x_{0}\right\|$.

Now, we are in a position to recall a fixed point theorem being an extension of results from [27].

Theorem 3. Let $E$ be a Banach space with $Q$ a nonempty, closed, convex, and weakly compact subset of $C[I, E]$. Assume that $T: Q \rightarrow Q$ is weakly-weakly sequentially continuous. Then $T$ has a fixed point in $Q$.

We need to introduce some subspaces of the space $P[I, E]$ of Pettis integrable functions on $I$ which are important in the sequel.

Definition 4. For $1 \leq p \leq \infty$, we define the class $\mathscr{H}^{p}(E)$ to be the class of all functions $x: I \rightarrow E$ having $\varphi x \in L^{p}(I)$ for every $\varphi \in E^{*}$. If $p=\infty$, the added condition

$$
\sup _{\|\varphi\|=1}(\operatorname{ess} \sup |\varphi x(t)|)<\infty
$$


must be satisfied by each $x \in \mathscr{H}^{\infty}(E)$. The class $\mathscr{H}_{0}^{p}(E)$ is defined by

$$
\mathscr{H}_{0}^{p}(E):=\left\{x \in P[I, E]: \varphi x \in L^{p}(I)\right\} .
$$

Remark 5. In a reflexive Banach space $E$ the set $\mathscr{H}_{0}^{1}(E)$ coincides with the space $P[I, E]$. This is due to the fact that in reflexive Banach spaces, the weakly measurable function $x$ : $I \rightarrow E$ is Pettis integrable if and only if $\varphi(x(\cdot))$ is Lebesgue integrable on $I$ for every $\varphi \in E^{*}$ [26]. In general, this is the space of Dunford integrable functions.

In the remaining part of this paper we let $p \in[1, \infty]$ be fixed and $q$ is conjugated with $p$; that is, $1 / p+1 / q=1$. The following results are due to Pettis (see [25, Theorem 3.4 and Corollary 3.41]).

Proposition 6. In order that $x(\cdot)$ could be in $\mathscr{H}_{0}^{p}(E)$, it is necessary and sufficient that $x(\cdot) u(\cdot)$ be Pettis integrable for every $u(\cdot) \in L^{q}(I)$.

It is worthwhile to recall the following.

Definition 7. Let $x: I \rightarrow E$. The (left-sided) fractional Pettisintegral (shortly LS-FPI) of $x$ of order $\alpha>0$ is defined by

$$
I_{+}^{\alpha} x(t):=\int_{0}^{t} \frac{(t-s)^{\alpha-1}}{\Gamma(\alpha)} x(s) d s, \quad t>0 .
$$

In the above definition the sign " " denotes the Pettis integral. For further purpose, we define the right-sided fractional Pettis-integral (shortly RS-FPI) by

$$
I_{-}^{\alpha} x(t):=\int_{t}^{1} \frac{(s-t)^{\alpha-1}}{\Gamma(\alpha)} x(s) d s, \quad t<1 .
$$

We will call a function fractionally Pettis integrable provided this integral exists as an element of $E$ (for arbitrary $t<1$ ).

We need to clarify the relations between Pettis integrability and fractional Pettis integrability. Similar results will be proved for classes $\mathscr{H}_{0}^{p}(E)$. This will be important in our consideration, but it seems to be really interesting in itself. Here we restrict ourselves to the case of left-sided fractional Pettis-integrals.

To make the paper more expository, we will consider fractional Pettis integrability for both cases: $\alpha<1$ and $\alpha>1$. The last case is more important in our paper, but the first one is necessary to compare our results with some earlier theorems.

Let us observe that such an integral $I_{+}^{\alpha} x(t):=\int_{0}^{t}((t-$ $\left.s)^{\alpha-1} / \Gamma(\alpha)\right) x(s) d s$ is a convolution of a function $h(\tau)=\tau^{\alpha-1} /$ $\Gamma(\alpha)$ for $\tau>0, h(\tau)=0$ for $\tau \leq 0$, and the function $(\widetilde{x})(t)=x(t)$ for $t \in I$, where $(\widetilde{x})(t)=0$ outside the interval $I$. Note that Pettis integrability of $x(t)$ implies Pettis integrability of $x(t+h)(h>0)$ and $x(-t)$, so the convolution of Pettis integrable function with real-valued function $h$ can be properly defined. We start with an obvious observation that for $\varphi \in E^{*}$

$$
\varphi\left(I_{+}^{\alpha} x\right)=\int_{0}^{t} \frac{(t-s)^{\alpha-1}}{\Gamma(\alpha)} \varphi x(s) d s .
$$

As a consequence of some properties of a convolution for the Pettis integral [28, Proposition 9], for arbitrary $\alpha$, we have the following.

Theorem 8. If $x: I \rightarrow E$ is Pettis integrable, then

(a) $I_{+}^{\alpha} x$ is defined almost a.e. on $I$,

(b) $x$ is fractionally Pettis integrable on $I$,

(c) if $x$ is Pettis integrable and strongly measurable, then $I_{+}^{\alpha} x: I \rightarrow E$ is bounded, weakly continuous and

$$
\sup _{\|\varphi\| \leq 1} \int_{0}^{1} \varphi I_{+}^{\alpha} x(t) d t \leq \sup _{\|\varphi\| \leq 1} \int_{0}^{1} \varphi x(t) d t \cdot\|h\|_{1} .
$$

In the case $E=\mathbb{R}$, it is a well-known consequence of an inequality of Young that the linear fractional integral operators $I_{+}^{\alpha}$, send $L^{q}([0,1])$ continuously into $L^{p}([0,1])$ if $p \in$ $[1, \infty]$ satisfy $q>1 /(\alpha+(1 / p))$ (see [29]) (a deep result from interpolation theory implies that even $q=1 /(\alpha+(1 / p))$ is allowed if $1<p<\infty)$. In particular, $I_{ \pm}^{\alpha}: L^{p}([0,1]) \rightarrow$ $L^{p}([0,1])$ is compact for each $p \in[1, \infty]$. Moreover, for $p>\max \{1,(1 / \alpha)\}$, the map $I_{ \pm}^{\alpha}: L^{p}([0,1]) \rightarrow C([0,1])$ is compact (see, e.g., $[20,30]$ ).

The following results plays a major rule in our analysis.

Lemma 9. For any $\alpha>0$ the operator $I_{ \pm}^{\alpha}$ takes $C\left[I, E_{w}\right]$ into $C\left[I, E_{w}\right]$ and is well defined.

Proof. Only the proof in case of the LS-FPI is given since the case of the RS-FPI is very similar.

It can be easily seen that if $x$ is weakly continuous, then $x \in \mathscr{H}_{0}^{\infty}(E)$. Since $s \rightarrow(t-s)^{\alpha-1} \in L^{1}, s<t$, in the view of Proposition 6 and Theorem 8 , we have that the function $s \rightarrow$ $(t-s)^{\alpha-1} x(s), s<t$, is Pettis integrable. Moreover, $I_{+}^{\alpha}$ is well defined. To see this, we define $y: I \rightarrow E$ by $y(t):=I_{+}^{\alpha} x(t)$, $t \in[0,1]$. From the definition of fractional Pettis integrals and Theorem 8(c), we have for every $\varphi \in E^{*}$ that

$$
\varphi y(t)=\int_{0}^{t} \frac{(t-s)^{\alpha-1}}{\Gamma(\alpha)} \varphi(x(s)) d s .
$$

Since the function $\varphi x$ is continuous, $\varphi y$ is continuous. That is, $y$ is weakly continuous which finishes the proof.

Lemma 10. Let $E$ be a reflexive Banach space. For any $\alpha \geq 1$ and arbitrary $p \in[1, \infty]$, the operator $I_{ \pm}^{\alpha}$ takes $\mathscr{H}_{0}^{p}(E)$ into $\mathscr{H}_{0}^{p}(E)$ and is well defined.

Proof. Only the proof in case of the LS-FPI is given since the case of the RS-FPI is very similar.

Note first that, for $x \in \mathscr{H}_{0}^{p}(E)$, we have in the view of Proposition 6 that the function $s \rightarrow(t-s)^{\alpha-1} x(s), s<t$, is 
Pettis integrable. That is, the operator $I^{\alpha}$ makes sense. Further, $I_{+}^{\alpha}$ is well defined. To see this, define $y: I \rightarrow E$ by $y(t):=$ $I_{+}^{\alpha} x(t), t \in[0,1]$. From the definition of fractional Pettis integrals, we have for every $\varphi \in E^{*}$ that

$$
\varphi(y(t))=\int_{0}^{t} \frac{(t-s)^{\alpha-1}}{\Gamma(\alpha)} \varphi(x(s)) d s=I_{+}^{1} I_{+}^{\alpha-1} \varphi(x(t)) .
$$

Since $x \in \mathscr{H}_{0}^{p}(E)$, one have $\varphi x \in L^{p}(I)$ for every $\varphi \in E^{*}$. One could see, by the properties of fractional integral operators on the Banach space $L^{p}(I)$, that $\varphi y \in L^{p}(I)$ for every $\varphi \in E^{*}$. In particular, $y$ is weakly continuous. Since weak continuity implies weak measurability (see [26, page 73]), $y(\cdot)$ is weakly measurable. However, in reflexive Banach spaces, weakly measurable functions $y: I \rightarrow E$ are Pettis integrable if and only if $y$ is Dunford integrable; that is, $\varphi(y(\cdot))$ is Lebesgue integrable on $I$ for every $\varphi \in E^{*}$. Thus $y(\cdot)$ is Pettis integrable. That is, $I_{+}^{\alpha} x \in \mathscr{H}_{0}^{p}(E)$.

Remark 11. Let us discuss some properties of $\mathscr{H}^{p}(E)$ and $\mathscr{H}_{0}^{p}(E)$. Recall that $h(\tau)=\tau^{\alpha-1} / \Gamma(\alpha)$ for $\tau>0$ and $z(\tau)=0$ for $\tau \leq 0$. The case of $\alpha \geq 1$ is trivial; that is, $h \in L^{\infty}(I)$. When $0<\alpha<1$, we see that $\int_{0}^{1}(z(s))^{q} d s=\int_{0}^{1}\left(s^{\alpha-1} / \Gamma(\alpha)\right)^{q} d s<\infty$ whenever $q<1 /(1-\alpha)$. This means that $z \in L^{1 /(1-\alpha)}(I)$ and by the converse for the Young inequality $I^{\alpha}$ takes $\mathscr{H}^{q_{1}}(E)$ into $\mathscr{H}^{s}(E)$ whenever $\left(1 / q_{1}\right)+(1 / q)=1+(1 / s)$. In particular, the space required for this property depends on $\alpha$. Let us note that $I^{\alpha}$ need not be continuous as an operator from $L^{1}(I)$ into $L^{1 /(1-\alpha)}(I)$ (cf. [29, Remark 4.1.1]).

This means that for $\alpha \geq 1$ we have "uniform" estimations for all $\alpha$, but for $0<\alpha<1$ the situation is more complicated (a weakly singular case).

Our consideration as well as Theorem 4.1.1 in [29] gives us a new property.

Lemma 12. For any $0<\alpha<1$ and arbitrary $1 \geq p \geq 1 / \alpha$ the operator $I_{ \pm}^{\alpha}$ takes $\mathscr{H}_{0}^{p}(E)$ into $\mathscr{H}_{0}^{s}(E)$, where $s=p /(1-p(\alpha-\varepsilon))$ with arbitrary $\varepsilon>0$.

As a consequence of Lemma 10, we are able to prove the following.

Lemma 13. Assume that $\alpha_{1}, \alpha_{2} \geq 1$ and $x \in \mathscr{H}_{0}^{p}(E)$. Then,

$$
I_{+}^{\alpha_{1}} I_{+}^{\alpha_{2}} x=I_{+}^{\alpha_{2}} I_{+}^{\alpha_{1}} x=I_{+}^{\alpha_{1}+\alpha_{2}} x
$$

Proof. As in the proof of Lemma 10 it follows that $I_{+}^{\alpha_{2}} x$ and $I_{+}^{\alpha_{1}+\alpha_{2}} x$ exist. By Lemma $10, I_{+}^{\alpha_{1}} I_{+}^{\alpha_{2}} x$ also exists. Therefore, for any $\varphi \in E^{*}$ we have

$$
\begin{aligned}
\varphi\left(I_{+}^{\alpha_{1}} I_{+}^{\alpha_{2}} x(t)\right) & =I_{+}^{\alpha_{1}} \varphi\left(I_{+}^{\alpha_{2}} x(t)\right) \\
& =I_{+}^{\alpha_{1}} I_{+}^{\alpha_{2}} \varphi(x(t)) \\
& =I_{+}^{\alpha_{1}+\alpha_{2}} \varphi(x(t)) \\
& =\varphi\left(I_{+}^{\alpha_{1}+\alpha_{2}} x(t)\right),
\end{aligned}
$$

that is,

$$
\varphi\left(I_{+}^{\alpha_{1}} I_{+}^{\alpha_{2}} x(t)-I_{+}^{\alpha_{1}+\alpha_{2}} x(t)\right)=0, \quad \text { for every } \varphi \in E^{*} .
$$

Hence $I_{+}^{\alpha_{1}} I_{+}^{\alpha_{2}} x=I_{+}^{\alpha_{1}+\alpha_{2}} x(t)$. Similarly, we are able to show that $I_{+}^{\alpha_{2}} I_{+}^{\alpha_{1}} x=I_{+}^{\alpha_{1}+\alpha_{2}} x$. This ends the proof.

Let us present the case $\alpha \geq 1$.

Lemma 14. If $\alpha \geq 1, \theta \in L^{q}[0,1]$, and $y \in \mathscr{H}_{0}^{p}(E)$, then

$$
\int_{0}^{1}\left[I_{-}^{\alpha} \theta(s)\right] y(s) d s=\int_{0}^{1} \theta(s)\left[I_{+}^{\alpha} y(s)\right] d s .
$$

Proof. Define the real-valued function $h$ by

$$
h(s):=\frac{1}{\Gamma(\alpha)} \int_{s}^{1}(\tau-s)^{\alpha-1} \theta(\tau) d \tau=I_{-}^{\alpha} \theta(s) .
$$

Using the properties of fractional calculus in the Banach space $L^{q}[0,1]$ (see, e.g., $[20,30]$ ), we deduce that $h \in L^{q}[0,1]$. Now, for $y \in \mathscr{H}_{0}^{p}(E)$ we have, in the view of Lemma 10, that $I_{+}^{\alpha} y \in \mathscr{H}_{0}^{p}(E)$. Thanks to Proposition 6, the functions $t \rightarrow$ $\theta(t) I_{+}^{\alpha} y(t)$ and $t \rightarrow h(t) y(t)$ are Pettis integrable on $[0,1]$. That is, the integrals in both sides of (13) exist. Then there exists $J \in E$, such that

$$
J=\int_{0}^{1} \theta(s) I_{+}^{\alpha} y(s) d s .
$$

By the definition of the Pettis integral, we have

$$
\begin{aligned}
\varphi J & =\int_{0}^{1} \theta(s) \varphi\left(I_{+}^{\alpha} y(s)\right) d s=\int_{0}^{1} \theta(s) I_{+}^{\alpha} \varphi y(s) d s \\
& =\int_{0}^{1} \theta(s) \int_{0}^{s} \frac{(s-\tau)^{\alpha-1}}{\Gamma(\alpha)} \varphi y(\tau) d \tau d s, \quad \forall \varphi \in E^{*} .
\end{aligned}
$$

By changing the order of integration results in

$$
\begin{aligned}
\varphi J & =\int_{0}^{1}\left(\int_{\tau}^{1} \frac{(s-\tau)^{\alpha-1}}{\Gamma(\alpha)} \theta(s) d s\right) \varphi y(\tau) d \tau \\
& =\int_{0}^{1} h(\tau) \varphi y(\tau) d \tau \\
& =\varphi\left(\int_{0}^{1} h(s) y(s) d s\right), \quad \forall \varphi \in E^{*} .
\end{aligned}
$$

Thus

$$
J=\int_{0}^{1} I_{-}^{\alpha} \theta(s) y(s) d s=\int_{0}^{1} u(s) I_{+}^{\alpha} y(s) d s .
$$

Definition 15. Let $x: I \rightarrow E$. We define the fractional pseudo-derivative of $x$ of arbitrary order $n+\alpha, \alpha \in[0,1)$, where $n \in \mathbb{N}_{0}:=\{0,1,2, \ldots\}$ by

$$
D^{n+\alpha} x(t):=D^{n+1} I_{+}^{1-\alpha} x(t),
$$

where $D$ denote the pseudo-differential operator (cf. [22, 25]). 
The following Lemma is well known in the case $E=\mathbb{R}$, but to see that it also holds in the vector-valued case, we provide a proof.

Lemma 16. For $0<\alpha \leq \beta$ we have for every weakly continuous function $x:[0,1] \rightarrow E, D^{0} x=x$, and

$$
D^{\beta} I_{+}^{\alpha} x=D^{\beta-\alpha} x
$$

In particular, when $\alpha=\beta$, (20) means that the operator $D^{\alpha} I_{+}^{\alpha}$ is defined in $C\left(I, E_{w}\right)$ and that $D^{\alpha}$ is the left-inverse of $I_{+}^{\alpha}$.

Proof. The first claim, that is, $D I_{+}^{1} x=x$, follows from the fact that the integral of weakly continuous function is weakly continuous, then pseudo-differentiable with respect to the right endpoint of the integration interval. Let $\beta=n+\gamma$ and $\beta-\alpha=m+\delta$ with $n, m \in \mathbb{N}_{0}$ and $\gamma, \delta \in[0,1)$. Then we have, in view of $D I^{1} x=x$ and Lemmas 9 and 10, that

$$
\begin{aligned}
D^{\beta} I_{+}^{\alpha} x & =D^{n+1} I_{+}^{1-\gamma} I_{+}^{\alpha} x \\
& =D^{n+1} I_{+}^{1-\gamma+\alpha} x \\
& =D^{n+1} I_{+}^{1+n-m-\delta} x \\
& =D^{m+1} D^{n-m} I_{+}^{n-m} I_{+}^{1-\delta} x \\
& =D^{m+1} I_{+}^{1-\delta} x \\
& =D^{\beta-\alpha} x .
\end{aligned}
$$

Definition 17. A function $u: I \rightarrow E$ is called pseudosolution of the problem (1) if $u \in C\left[I, E_{w}\right]$ has fractional pseudo-derivative of order $\alpha \in(1,2], u(0)=0, u(1)+$ $\int_{0}^{1} \mathfrak{\Im}(\tau) u(\tau) d \tau=l$ and satisfies

$$
\begin{array}{r}
D^{2} \varphi\left(I_{+}^{2-\alpha} u(t)\right)+\varphi\left(f\left(t, u(t), D^{\beta} u(t)\right)\right)=0 \\
\text { a.e. on }[0,1], \text { for each } \varphi \in E^{*}
\end{array}
$$

The following auxiliary Lemma will be needed in our techniques.

Lemma 18. If $v \in C\left[I, E_{w}\right]$ is a pseudo-solution to the problem

$$
\begin{gathered}
D^{\alpha-\beta} v(t)+f\left(t, I_{+}^{\alpha} v(t), v(t)\right)=0, \\
t \in[0,1], \quad \alpha \in(1,2], \quad \beta \in(0,1], \quad \alpha>1+\beta, \\
I_{+}^{\beta} v(1)+\int_{0}^{1} \Im(\tau) I_{+}^{\beta} v(\tau) d \tau=l, \quad v(0)=0,
\end{gathered}
$$

then $u:=I_{+}^{\beta} v$ is a pseudo-solution for the problem (1).

Proof. Let $v \in C\left[I, E_{w}\right]$ be a pseudo-solution to the problem (23) and $\varphi \in E^{*}$. As in the proof of Lemma 9 it follows that
$I_{+}^{\beta} v$ exists and the real function $\varphi u$ is continuous for every $\varphi \in E^{*}$; moreover

$$
\begin{aligned}
\lim _{t \rightarrow 0^{+}} \varphi u(t) & =\lim _{t \rightarrow 0^{+}}\left(I_{+}^{\beta} \varphi v\right)(t) \\
& =\lim _{t \rightarrow 0^{+}} \int_{0}^{t} \frac{(t-s)^{\beta-1}}{\Gamma(\beta)} \varphi v(s) d s=0 .
\end{aligned}
$$

Thus $\varphi u(0)=0$ for every $\varphi \in E^{*}$; that is, $u(0)=0$. Further

$$
u(1)+\int_{0}^{1} \mathfrak{I}(\tau) u(\tau) d \tau=l
$$

In the view of Lemma 16 we also have

$$
D^{\alpha} u(t)=\left(D^{\alpha} I_{+}^{\beta} v\right)(t)=D^{\alpha-\beta} v(t)
$$

If otherwise is not stated, we will assume from now that $\alpha \in(1,2]$ and $\beta \in(0,1]$.

To obtain the Hammerstein type integral equation modeled off the problem (23), we keep the boundary value problem (23) in mind and we formally put (cf. [11, Lemma 2.3])

$$
v(t)=-I_{+}^{\alpha-\beta} f\left(t, I_{+}^{\beta} v(t), v(t)\right)+c t^{\alpha-\beta-1} .
$$

In the view of Lemma 13, we obtain

$$
I_{+}^{\beta} v(t)=-I_{+}^{\alpha} f\left(t, I_{+}^{\beta} v(t), v(t)\right)+c\left[\frac{\Gamma(\alpha-\beta)}{\Gamma(\alpha)}\right] t^{\alpha-1}
$$

To facilitate our discussion, let $q \in[1, \infty]$ be constant with the conjugate exponents $p$. Suppose $\mathfrak{J} \in L^{q}[0,1]$ be a nonnegative real-valued function and $f:[0,1] \times E \times E \rightarrow E$ satisfy the following assumptions:

(1) for each $t \in I=[0,1], f(t, \cdot, \cdot):(I) \times E \times E \rightarrow E$ is weakly-weakly sequentially continuous,

(2) for each $x, y \in C\left(I, E_{w}\right), f(\cdot, x(\cdot), y(\cdot)) \in \mathscr{H}_{0}^{p}(E)$,

(3) for any $r>0$ and $\varphi \in E^{*}$ there exist a Pettis integrable function $\tilde{f}: I \rightarrow E$, function $\Psi \in L^{p}\left[I, \mathbb{R}^{+}\right]$, and nondecreasing continuous function $\Omega:[0, \infty) \rightarrow$ $(0, \infty)$ such that $|\varphi(f(t, x, y))| \leq|\varphi(\tilde{f}(t))| \Omega(r) \leq$ $\|\varphi\| \Psi(t) \Omega(r)$ for a.e. $t \in I$ and all $(x, y) \in B_{r} \times B_{r}$.

Let us present two remarks about the above assumptions.

(i) For the interesting discussion about the growth conditions for Pettis integrable functions of the above type see [31]. For differential equations with Caputo fractional integrals (i.e., solutions in the space $C^{m}(0$, $1), \mathbb{R}^{d}$ ), where $m-1<\alpha<m$ the problem of dominants for considered functions (Assumption (3)) was considered in [32]. Nevertheless, in the paper by Lin rather strong boundedness conditions are investigated (dominants from $L^{2}(I)$ or $L^{4}(I)$ for $\alpha>$ $1 / 2$ or bounded functions for $\alpha>0$ ) [32, Remark 2.3]. 
(ii) For convenience of the readers, let us recall the following lemma describing particular sufficient conditions for Pettis integrability of $f(\cdot, x(\cdot), y(\cdot))[23$, Lemma 15]. It is obvious that additional growth condition for $f$ allows us to characterize the functions from $\mathscr{H}_{0}^{p}(E)$.

Lemma 19. Assume that $x$ is absolutely continuous and $f$ : $I \times E \times E \rightarrow E$. Thus $f(\cdot, x(\cdot), y(\cdot))$ is Pettis integrable if at least one of the following cases holds:

(a) $f$ satisfies Carathédory conditions; that is, $f(\cdot, x, y)$ is measurable, $f(t, \cdot, \cdot)$ is continuous in $E \times E$, and there exists an integrable function $h: I \rightarrow \mathbb{R}$ such that $\|f(t, x, y)\| \leq h(t)$ for all $x, y \in E$ and a.e. $t \in I$,

(b) $f$ is weakly-weakly continuous and $E$ is a weakly sequentially complete space,

(c) $f(\cdot, x, y)$ is weakly measurable, $f(t, \cdot, \cdot)$ is weaklyweakly continuous in $E \times E$ and $E$ is a WCG-space (weakly compactly generated space),

(d) $f$ is strongly measurable and there exists a Young function $\Gamma$ such that $\lim _{x \rightarrow \infty} \Gamma(x) / x=+\infty$ and $\varphi f \epsilon$ $L^{\Gamma}(I)$,

(e) $f$ is strongly measurable and there exists $p>1$ such that $\varphi f \in L^{p}$ for each $\varphi \in E^{*}$ (here $f(\cdot, x(\cdot), y(\cdot)) \in$ $\left.\mathscr{H}_{0}^{p}(E)\right)$,

(f) $f(\cdot, x, y)$ is strongly measurable, $f(t, \cdot, \cdot)$ is weaklyweakly sequentially continuous in $E \times E$, and $f$ is bounded,

(g) $f(\cdot, x(\cdot), y(\cdot))$ is strongly measurable, E contains no copy of $c_{0}$, and $f$ is bounded. $c$ by

Now, we would like to pay our attention to solve (27) for

$$
I_{+}^{\beta} v(1)+\int_{0}^{1} \mathfrak{I}(\tau) I_{+}^{\beta} v(\tau) d \tau=l
$$

It follows that

$$
\begin{aligned}
& c\left[\frac{\Gamma(\alpha-\beta)}{\Gamma(\alpha)}\right]-\int_{0}^{1} \frac{(1-s)^{\alpha-\beta-1} f\left(s, I_{+}^{\beta} v(s), v(s)\right)}{\Gamma(\alpha-\beta)} d s=l, \\
& -\int_{0}^{1} \mathfrak{\Im}(\tau)\left(c\left[\frac{\Gamma(\alpha-\beta)}{\Gamma(\alpha)}\right] \tau^{\alpha-\beta-1}\right. \\
& \left.-\int_{0}^{\tau} \frac{(\tau-s)^{\alpha-\beta-1} f\left(s, I_{+}^{\beta} v(s), v(s)\right)}{\Gamma(\alpha-\beta)} d s\right) d \tau \\
& =l-c\left[\frac{\Gamma(\alpha-\beta)}{\Gamma(\alpha)}\right] \int_{0}^{1} \mathfrak{\Im}(\tau) \tau^{\alpha-\beta-1} d \tau \\
& +\int_{0}^{1} \mathfrak{J}(\tau)\left(\int_{0}^{\tau} \frac{(\tau-s)^{\alpha-\beta-1} f\left(s, I_{+}^{\beta} v(s), v(s)\right)}{\Gamma(\alpha-\beta)} d s\right) d \tau .
\end{aligned}
$$

Therefore

$$
\begin{aligned}
& c\left[\frac{\Gamma(\alpha-\beta)}{\Gamma(\alpha)}\right](1+\gamma) \\
& =l+\int_{0}^{1} \mathfrak{J}(\tau)\left(\int_{0}^{\tau} \frac{(\tau-s)^{\alpha-\beta-1} f\left(s, I_{+}^{\beta} v(s), v(s)\right)}{\Gamma(\alpha-\beta)} d s\right) d \tau \\
& +\int_{0}^{1} \frac{(1-s)^{\alpha-\beta-1} f\left(s, I_{+}^{\beta} v(s), v(s)\right)}{\Gamma(\alpha-\beta)} d s,
\end{aligned}
$$

where

$$
\gamma=\int_{0}^{1} \mathfrak{I}(\tau) \tau^{\alpha-\beta-1} d \tau
$$

Then (in account of Lemma 14), we have

$$
\begin{aligned}
c= & \frac{\Gamma(\alpha)}{\Gamma(\alpha-\beta)(1+\gamma)} \\
& \times\left[l+\int_{0}^{1} \frac{(1-s)^{\alpha-\beta-1} f\left(s, I_{+}^{\beta} v(s), v(s)\right)}{\Gamma(\alpha-\beta)} d s\right. \\
& \left.\quad+\int_{0}^{1} h(s) f\left(s, I_{+}^{\beta} v(s), v(s)\right) d s\right] .
\end{aligned}
$$

Here $h=I_{-}^{\alpha-\beta} \mathfrak{\Im}$. Substituting $c$ into (27), one has

$$
\begin{aligned}
v(t)= & -I_{+}^{\alpha-\beta} f\left(t, I_{+}^{\beta} v(t), v(t)\right)+\frac{l \Gamma(\alpha) t^{\alpha-\beta-1}}{\Gamma(\alpha-\beta)(1+\gamma)} \\
& +\frac{\Gamma(\alpha) t^{\alpha-\beta-1}}{\Gamma(\alpha-\beta)(1+\gamma)} \\
& \times \int_{0}^{1}\left[\frac{(1-s)^{\alpha-\beta-1}}{\Gamma(\alpha-\beta)}+h(s)\right] f\left(s, I_{+}^{\beta} v(s), v(s)\right) d s \\
= & \frac{l \Gamma(\alpha) t^{\alpha-\beta-1}}{\Gamma(\alpha-\beta)(1+\gamma)} \\
& +\int_{0}^{t}\left[\frac{\Gamma(\alpha) \frac{(t(1-s))^{\alpha-\beta-1}}{(1+\gamma)(\Gamma(\alpha-\beta))^{2}}}{\Gamma(t-s)^{\alpha-\beta-1}}\right] f\left(s, I_{+}^{\beta} v(s), v(s)\right) d s \\
& +\int_{t}^{1} \Gamma(\alpha) \frac{(t(1-s))^{\alpha-\beta-1}}{(1+\gamma)(\Gamma(\alpha-\beta))^{2}} \\
& \times f\left(s, I_{+}^{\beta} v(s), v(s)\right) d s \\
& \quad \frac{\Gamma(\alpha) t^{\alpha-\beta-1}}{\Gamma(\alpha-\beta)(1+\gamma)} \int_{0}^{1} h(s) f\left(s, I_{+}^{\beta} v(s), v(s)\right) d s .
\end{aligned}
$$


Thus

$$
v(t)=p(t)+\int_{0}^{1} G(t, s) f\left(s, I_{+}^{\beta} v(s), v(s)\right) d s, \quad t \in[0,1],
$$

where $p(t)=l \Gamma(\alpha) t^{\alpha-\beta-1} / \Gamma(\alpha-\beta)(1+\gamma)$. The Green function $G$ is given by $G(t, s)=G_{1}(t, s)+G_{2}(t, s)$ with

$$
\begin{aligned}
& G_{1}(t, s) \\
& := \begin{cases}\frac{\Gamma(\alpha)}{\Gamma(\alpha-\beta)}\left[\frac{(t(1-s))^{\alpha-\beta-1}}{(1+\gamma) \Gamma(\alpha-\beta)}-\frac{(t-s)^{\alpha-\beta-1}}{\Gamma(\alpha)}\right], & 0 \leq s \leq t \leq 1, \\
\frac{\Gamma(\alpha)}{\Gamma(\alpha-\beta)}\left[\frac{(t(1-s))^{\alpha-\beta-1}}{(1+\gamma) \Gamma(\alpha-\beta)}\right], & 0 \leq t \leq s \leq 1,\end{cases} \\
& G_{2}(t, s):=\frac{\Gamma(\alpha) t^{\alpha-\beta-1}}{\Gamma(\alpha-\beta)(1+\gamma)} h(s), \quad t, s \in[0,1] .
\end{aligned}
$$

Since $\alpha-\beta>1$, the following can be easily seen.

Lemma 20. The mapt $\rightarrow G(t, \cdot)$ is continuous from $[0,1]$ to $L^{q}[0,1]$.

Remark 21. We point out that if $E$ is reflexive, it is not necessary to assume any compactness conditions on the nonlinearity of $f$. This will be due to [33, Lemma 2] and the fact that a subset of reflexive Banach spaces is weakly compact if and only if it is weakly closed and norm bounded.

\section{Weak Solutions of the Hammerstein Integral Equation}

In this section, in the light of the Assumptions (1)-(3) imposed on $f$, we proceed to obtain a result which relies on the fixed point Theorem 3 to ensure the existence of weak solution to the integral equation (35). For the sake of convenience, we introduce the following.

Definition 22. By a solution to (35) we mean a function $v \in C(I, E)$ which satisfies the integral equation (35). This is equivalent to the finding $v \in C(I, E)$ with

$$
\begin{array}{r}
\varphi(v(t))=\varphi\left(p(t)+\int_{0}^{1} G(t, s) f\left(s, I_{+}^{\beta} v(s), v(s)\right) d s\right), \\
t \in I \forall \varphi \in E^{*}
\end{array}
$$

We need to explain why we consider continuous solutions. By the properties of the Pettis integral this should be weakly continuous function. Since $p$ is continuous and we impose (local) boundedness hypothesis for $f$, our solutions are strongly continuous (cf. [22]). We restrict our attention to the space $C(I, E)$ and then our integral operators will be defined on this space. In contrast to the case of weakly-weakly continuous functions $f$, we need to replace the space $C\left(I, E_{w}\right)$ endowed with its topology of weak uniform convergence by the space of (strongly) continuous functions $C(I, E)$ with its weak topology. We will utilize in our proofs some characterization of its weak topology.

Now, we are in the position to state and prove the first existence result.

Theorem 23. Assume that $\alpha \in(1,2], \beta \in(0,1)$ with $\alpha>1+\beta$ and $\mathfrak{J} \in L^{q}([0,1])$ be a nonnegative real-valued function. If the Assumptions (1)-(3) hold along with

$$
\left(\sup _{t \in[0,1]} \int_{0}^{1}|G(t, s)| \Psi(s) d s\right) \limsup _{r \rightarrow \infty} \frac{\Omega(r)}{r}<\Gamma(1+\beta),
$$

then the integral equation (35) has at least one solution $v \in$ $C[I, E]$.

Proof. First of all, observe the expression of $G$ and note that the following implications:

$$
\begin{array}{r}
\Im \in L^{q}[0,1] \Longrightarrow h \in L^{q}[0,1] \Longrightarrow G(t, \cdot) \in L^{q}[0,1], \\
t \in[0,1],
\end{array}
$$

hold. Consequently $G(t, \cdot) \Psi(\cdot) \in L^{1}[0,1]$ for any $t \in[0,1]$. Let

$$
\rho=\left(\sup _{t \in[0,1]} \int_{0}^{1}|G(t, s)| \Psi(s) d s\right) \limsup _{r \rightarrow \infty} \frac{\Omega(r)}{r} .
$$

Consider the set $S$ of real numbers $r \geq 0$ which satisfy the inequality

$$
r \Gamma(1+\beta) \leq\|p\|_{0}+\Omega(r)\left(\sup _{t \in[0,1]} \int_{0}^{1}|G(t, s)| \Psi(s) d s\right) .
$$

Then $S$ is bounded above; that is, there exists a constant $R_{0}$ with

$$
r \leq R_{0} \quad \forall r \in S .
$$

To see this, suppose (42) is false. Then there exists a sequence $0 \neq r_{n} \in S$ with $r_{n} \rightarrow \infty$ as $n \rightarrow \infty$ and

$$
\Gamma(1+\beta) \leq \frac{\|p\|_{0}}{r_{n}}+\frac{\Omega\left(r_{n}\right)}{r_{n}}\left(\sup _{t \in[0,1]} \int_{0}^{1}|G(t, s)| \Psi(s) d s\right) .
$$

Since $\lim \sup \left(s_{n}+t_{n}\right) \leq \lim \sup s_{n}+\lim \sup t_{n}$ for any sequences $s_{n} \geq 0, t_{n} \geq 0$, we have $\rho \geq \Gamma(1+\beta)$. This contradicts (38). Then, for every $R>R_{0}$ the inequality

$$
\|p\|_{0}+\Omega(R)\left(\sup _{t \in[0,1]} \int_{0}^{1}|G(t, s)| \Psi(s) d s\right)<\Gamma(1+\beta) R
$$

holds, which is in contradiction with $R \in S$ and then contradicts (42). 
Now, define the operator $T: C[I, E] \rightarrow C[I, E]$ by

$$
T v(t):=p(t)+\int_{0}^{1} G(t, s) f\left(s, I_{+}^{\beta} v(s), v(s)\right) d s, \quad t \in[0,1] .
$$

We remark that for $v \in C[I, E]$ we have that, by Lemma 9, $I_{+}^{\beta} v$ is weakly continuous, and consequently, $f\left(\cdot, I_{+}^{\beta} v(\cdot), v(\cdot)\right) \in$ $\mathscr{H}_{0}^{p}(E)$ (Assumption (2)). Since $s \mapsto G(t, s) \in L^{q}(I)$, for all $t \in[0,1], G(t, \cdot) f\left(\cdot, I_{-}^{\beta} v(\cdot), v(\cdot)\right)$ is Pettis integrable for all $t \in[0,1]$ (thanks to Proposition 6), and thus the operator $T$ makes sense. Note that $T$ is well defined. To see this, let $t_{1}, t_{2} \in[0,1]$ with $t_{2}>t_{1}$. Since $\beta \in(0,1)$, we deduce that if $\|v\| \leq \sigma_{1}$, then $\|v\|<\sigma$ and $\left\|I_{+}^{\beta} v\right\| \leq \sigma$, where $\sigma=\sigma_{1} / \Gamma(1+\beta)$. Without loss of generality, assume $T v\left(t_{2}\right)-T v\left(t_{1}\right) \neq 0$. Then there exists (as a consequence of Proposition 2 ) $\varphi \in E^{*}$ with $\|\varphi\|=1$ and $\left\|T v\left(t_{2}\right)-T v\left(t_{1}\right)\right\|=\varphi\left(T v\left(t_{1}\right)-T v\left(t_{1}\right)\right)$.

Putting the Assumption (3) in mind, one can write the following chain of inequalities:

$$
\begin{array}{rl}
\| T & v\left(t_{2}\right)-T v\left(t_{1}\right) \| \\
= & \varphi\left(T v\left(t_{2}\right)-T v\left(t_{1}\right)\right) \\
\leq & \varphi\left(p\left(t_{2}\right)-p\left(t_{1}\right)\right) \\
& +\Omega(\sigma) \int_{0}^{1}\left|G\left(t_{2}, s\right)-G\left(t_{1}, s\right)\right| \Psi(s) d s \\
\leq & \left\|p\left(t_{2}\right)-p\left(t_{1}\right)\right\| \\
& +\Omega(\sigma)\left(\int_{0}^{1}\left|G\left(t_{2}, s\right)-G\left(t_{1}, s\right)\right|^{q} d s\right)^{1 / q} \\
& \times\left(\int_{0}^{1}|\Psi(s)|^{p} d s\right) \\
\leq & \left\|p\left(t_{2}\right)-p\left(t_{1}\right)\right\|+\Omega(\sigma)\left\|G\left(t_{2}, \cdot\right)-G\left(t_{1}, \cdot\right)\right\|_{q}\|\Psi\|_{p} \\
\leq & \left\|p\left(t_{2}\right)-p\left(t_{1}\right)\right\| \\
& +\Omega(\sigma)\left(\left\|G_{1}\left(t_{2}, \cdot\right)-G_{1}\left(t_{1}, \cdot\right)\right\|_{q}\right. \\
\leq & \left.+\left\|G_{2}\left(t_{2}, \cdot\right)-G_{2}\left(t_{1}, \cdot\right)\right\|_{q}\right)\|\Psi\|_{p}
\end{array}
$$

Then

$$
\begin{aligned}
& \left\|T v\left(t_{2}\right)-T v\left(t_{1}\right)\right\| \\
& \leq\left\|p\left(t_{2}\right)-p\left(t_{1}\right)\right\| \\
& +\Omega(\sigma)\left(\left\|G_{1}\left(t_{2}, \cdot\right)-G_{1}\left(t_{1}, \cdot\right)\right\|_{q}\right. \\
& \left.+\frac{\Gamma(\alpha)\left|t_{2}^{\alpha-\beta-1}-t_{1}^{\alpha-\beta-1}\right|\|h\|_{q}}{\Gamma(\alpha-\beta)(1+\gamma)}\right)\|\Psi\|_{p} .
\end{aligned}
$$

Therefore we deduce, in the view of Lemma 20, that $T$ maps $C[I, E]$ into itself.

Let $Q \in C[I, E]$ be the convex, closed and equicontinuous subset (required by Theorem 3). Define this set by

$$
\begin{aligned}
& Q:=\left\{v \in C[I, E]:\|v\|_{0} \leq R_{0},\right. \\
& \forall t_{1}, t_{2} \in[0,1] \text { we have }\left\|v\left(t_{2}\right)-v\left(t_{1}\right)\right\| \\
& \leq\left\|p\left(t_{2}\right)-p\left(t_{1}\right)\right\| \\
& +\Omega\left(\frac{R_{0}}{\Gamma(1+\beta)}\right) \\
& \times\left(\left\|G_{1}\left(t_{2}, \cdot\right)-G_{1}\left(t_{1}, \cdot\right)\right\|_{q}\right. \\
& \left.\left.+\frac{\Gamma(\alpha)\left|t_{2}^{\alpha-\beta-1}-t_{1}^{\alpha-\beta-1}\right|\|h\|_{q}}{\Gamma(\alpha-\beta)(1+\gamma)}\right)\|\Psi\|_{p}\right\} .
\end{aligned}
$$

We claim that $T$ restricted to the set $Q$ maps this set into itself (i.e., $T: Q \rightarrow Q$ ) and is weakly-weakly sequentially continuous. Once the claim is established, Theorem 3 guarantees the existence of a fixed point of $T$. Hence the integral equation (35) has a solution in $C[I, E]$.

We start by showing that $T: Q \rightarrow Q$. To see this, take $v \in Q, t \in[0,1]$. Since $\beta \in(0,1)$, we deduce that $\|v\| \leq R_{0}<$ $R_{0} / \Gamma(1+\beta),\left\|I^{\beta} v\right\| \leq R_{0} / \Gamma(1+\beta)$. The monotonicity of $\Omega$ and the inequality (47) imply that

$$
\begin{aligned}
& \left\|T v\left(t_{2}\right)-T v\left(t_{1}\right)\right\| \\
& \leq\left\|p\left(t_{2}\right)-p\left(t_{1}\right)\right\| \\
& +\Omega\left(\frac{R_{0}}{\Gamma(1+\beta)}\right) \\
& \times\left(\left\|G_{1}\left(t_{2}, \cdot\right)-G_{1}\left(t_{1}, \cdot\right)\right\|_{q}\right. \\
& \left.\quad+\frac{\Gamma(\alpha)\left|t_{2}^{\alpha-\beta-1}-t_{1}^{\alpha-\beta-1}\right|\|h\|_{q}}{\Gamma(\alpha-\beta)(1+\gamma)}\right)\|\Psi\|_{p} .
\end{aligned}
$$


Now, without loss of generality, assume $T v(t) \neq 0$. Then there exists (consequence of Proposition 2) $\varphi \in E^{*}$ with $\|\varphi\|=1$ and $\|T x(t)\|=\varphi(T v(t))$. By the Assumption (3), we obtain

$$
\begin{aligned}
& \|T v(t)\| \\
& \quad \leq \varphi(p(t))+\varphi\left(\int_{0}^{1} G(t, s) f\left(s, I_{+}^{\beta} v(s), v(s)\right) d s\right) \\
& \quad \leq\|p(t)\|+\sup _{t \in[0,1]} \int_{0}^{1}|G(t, s)| \cdot\left|\varphi f\left(s, I_{+}^{\beta} v(s), v(s)\right)\right| d s \\
& \quad \leq\|p\|_{0}+\Omega\left(\frac{R_{0}}{\Gamma(1+\beta)}\right)\left(\sup _{t \in[0,1]} \int_{0}^{1} G(t, s) \Psi(s) d s\right) \\
& \quad<\frac{R_{0}}{\Gamma(1+\beta)} \Gamma(1+\beta)=R_{0} .
\end{aligned}
$$

Therefore $\|T v\|_{0}=\sup _{t \in[0,1]}\|T v(t)\| \leq R_{0}$. Hence $T: Q \rightarrow Q$.

We need to prove now that $T: Q \rightarrow Q$ is weaklyweakly sequentially continuous. Let us recall that the weak convergence in $Q \subset C(I, E)$ is exactly the weak pointwise convergence. Let $\left(v_{n}\right)$ be a sequence in $Q$ weakly convergent to $v$. Then $v_{n}(t) \rightarrow v(t)$ in $E_{w}$ for each $t \in[0,1]$. Since $Q$ is closed, by Lemma 1 we have $v \in Q$.

Fix $t \in I$ and note, in the view of Lebesgue dominated convergence theorem for the Pettis integral (see $[31,34])$, that $I_{+}^{\beta} v_{n}(s) \rightarrow I_{+}^{\beta} v(s)$ in $E_{w}$. Let us recall that the topology on $C\left(I, E_{w}\right)$ on equicontinuous subsets coincides with the topology of weak pointwise convergence. Since $f$ satisfies Assumption (1), we have $f\left(t, I_{-}^{\beta} v_{n}(t), v_{n}(t)\right)$ converging weakly to $f\left(t, I_{-}^{\beta} v(t), v(t)\right)$; hence again the Lebesgue dominated convergence theorem for Pettis integral yields $T v_{n}(t)$ converging weakly to $T v(t)$ in $E$, but $Q$ is an equicontinuous subset of $C(I, E)$, and then $T: Q \rightarrow Q$ is weakly-weakly sequentially continuous. Applying now Theorem 3, we conclude that $T$ has a fixed point in $Q$, which completes the proof.

Let us present a multivalued problem:

$$
\begin{gathered}
D^{\alpha-\beta} v(t) \in F\left(t, I_{+}^{\alpha} v(t), v(t)\right), \\
t \in[0,1], \quad \alpha \in(1,2], \quad \beta \in(0,1], \quad \alpha>1+\beta, \\
I_{+}^{\beta} v(1)+\int_{0}^{1} \mathfrak{I}(\tau) I_{+}^{\beta} v(\tau) d \tau=l, \quad v(0)=0 .
\end{gathered}
$$

Some basic results for multivalued boundary value problems with Pettis integrals are due to Maruyama [35], Azzam et al. [36], Azzam-Laouir and Boutana [37], and Satco [38]. However these results are devoted to study the standard case $\alpha-\beta=2$ and three-point boundary conditions. Our result is an essential extension for the previous ones.

By $c k(E)$ and $c w k(E)$ we denote the family of all nonempty convex compact and nonempty convex weakly compact subsets of $E$, respectively. For every nonempty convex bounded set $C \subset E$ the support function of $C$ is denoted by $s(\cdot, C)$ and defined on $E^{*}$ by $s(\varphi, C)=\sup _{x \in C} \varphi x$, for each $\varphi \in E^{*}$.
Definition 24. A multifunction $F: E \rightarrow 2^{E}$ with nonempty, closed values is weakly sequentially upper hemicontinuous if and only if for each $\varphi \in E^{*} s(\varphi, G(\cdot)): E \rightarrow \mathbb{R}$ is sequentially upper semicontinuous from $(E, w)$ into $\mathbb{R}$.

In the remaining part of the paper a multifunction $F$ is supposed to be Pettis integrable in the sense of Aumann.

Definition 25. The Aumann-Pettis integral of a multifunction $F: I \rightarrow E$ is

$$
I_{A}:=(A P) \int_{I} F(s) d s=\left\{(P) \int_{I} f(s) d s: f \in S_{F}^{P e}\right\},
$$

where $S_{F}^{P e}$ denotes the set of all Pettis integrable selections of $F$ provided that this set is not empty.

Let us note that the multivalued Pettis integral can be defined by other methods. The above definition is the best choice for our consideration. This can be deduced from the following theorem.

Theorem 26 (see [39]). Let $F: I \rightarrow c w k(E)[c k(E)]$ be measurable and scalarly integrable multifunction (i.e., the support functions are real-valued integrable functions). Then the following statements are equivalent:

(a) the set $\left\{\varphi f: \varphi \in B\left(E^{*}\right), f \in S_{F}^{P e}\right\}$ is uniformly integrable,

(b) every measurable selection of $F$ is Pettis integrable,

(c) for every measurable subset A of I the Aumann-Pettis integral $I_{A}$ belongs to $c w k(E)[c k(E)]$ and, for every $\varphi \in$ $E^{*}$, one has

$$
s\left(\varphi, I_{A}\right)=\int_{A} s(\varphi, F(s)) d s .
$$

Taking into account Theorem 8 , we are able to add one more condition to the above theorem, which seems to be important in our consideration. Since $\alpha-\beta>1, G$ is continuous, and by taking arbitrary Pettis integrable selection we obtain Pettis integrability of $G(t, \cdot) f(\cdot)$.

Theorem 27. Each of the conditions from Theorem 26 implies the following:

(d) for every the multifunction $F$ is fractionally AumannPettis integrable; that is, $I_{+}^{\alpha} F(t):=\left\{\int_{0}^{t}\left((t-s)^{\alpha-1} /\right.\right.$ $\left.\Gamma(\alpha)) f(s) d s, f \in S_{F}^{P e}\right\}$ belongs to $c w k(E)[c k(E)]$.

Let us recall that we restrict ourselves to the case of the (left-sided) fractional Pettis-integral.

Note that for multivalued mappings we will utilize Kakutani's fixed point theorem (for continuity concepts see [27]).

Theorem 28 (see [27]). If $C$ is a nonempty weakly compact convex subset of $E$ and $F: C \rightarrow 2^{C}$ is sequentially weakly upper semi-continuous, then there exists a fixed point of $F$; that is, $x \in C$ with $x \in F(x)$. 
An immediate consequence of the above theorems as well as our main theorems is the following result.

Theorem 29. Assume that $E$ is separable. Let $F: I \times E \times E \rightarrow$ $2^{E}$ with nonempty convex and weakly compact values satisfy the following:

(a) $F(t, \cdot, \cdot): E \times E \rightarrow E$ is weakly sequentially upper hemicontinuous for each $t \in I$,

(b) $F(\cdot, x, y)$ has a weakly measurable selection for each $(x, y) \in E \times E$,

(c) $F(t, x, y) \subset H(t)$ a.e. for some cwk-(E-) valued Pettis integrable multifunction $G$.

Then there exists at least one pseudo-solution of the Cauchy problem (51) on I.

We will follow the idea of the proof for the single-valued problem. Let us only sketch the main steps of the proof.

Note that the Assumption (c) implies weak compactness of $S_{H}^{P e}$ and separability of the space $E$ are sufficient to replace reflexivity of $E$ (as announced in the preliminary part).

In this proof we need to define the multifunction $T$ : $C[I, E] \rightarrow 2^{C[I, E]}$ by

$$
T v(t):=p(t)+\int_{0}^{1} G(t, s) F\left(s, I_{+}^{\beta} v(s), v(s)\right) d s,
$$

$$
t \in(I)
$$

By [40, Lemma 3.2] our assumptions (a)-(c) imply that the set $S_{F}^{P e}(\cdot, v(\cdot))$ is nonempty for arbitrary $v \in C(I, E)$ and the multivalued Nemytskii operator is well defined, so by Theorem 27 the Aumann-Pettis integral of $F$ is nonempty too.

Let $W=\left\{f \in \mathscr{H}_{0}^{1}(E): f(t) \in H(t)\right.$ a.e. on $\left.I\right\}$ and $U=$ $\left\{x_{f} \in C(I, E): x_{f}(t)=p(t)+\int_{0}^{t} G(t, s) f(s) d s, t \in I, f \in W\right\}$.

For $f \in W$ and $\varphi \in E^{*}$ we have $\varphi f \leq s(\varphi, H)$. Then, by our assumptions, $W$ is Pettis uniformly integrable. Thus for arbitrary $x \in U$ and $t, \tau \in I$ there exists an appropriate $f \in W$ and

$$
\begin{aligned}
\|x(t)-x(\tau)\| & =\sup _{\|\varphi\| \leq 1} \varphi(x(t)-x(\tau)) \\
& =\sup _{\|\varphi\| \leq 1}\left(\int_{0}^{t} \varphi f(s) d s-\int_{0}^{\tau} \varphi f(s) d s\right) \\
& =\sup _{\|\varphi\| \leq 1} \int_{\tau}^{t} \varphi f(s) d s \\
& \leq \sup _{\|\varphi\| \leq 1} \int_{\tau}^{t} s(\varphi, H(s)) d s .
\end{aligned}
$$

By uniform Pettis integrability of $W$ it follows that $U$ is an equicontinuous subset of $C(I, E)$. The property of the multivalued Pettis integral gives us the convexity of $U$. Then $U$ is nonempty, convex, bounded, and equicontinuous in $C(I, E)$.
As $S_{H}^{P e}$ is sequentially compact for the topology induced by the tensor product $L^{\infty} \otimes E^{*}$, the $\bar{U}$ is closed. Since $U$ is convex, by Mazur's lemma (Lemma 1) $\bar{U}$ is weakly closed. Thus by a weak version of Ascoli's theorem $\bar{U}$ is weakly compact in $C(I, E)$.

As the set $U$ is strongly equicontinuous, then for each $M>0$ there exists $\alpha \in I$ such that for each $t \in I$ and $f \in S_{F}^{P e}$ we have $\left\|\int_{0}^{t} f(s) d s\right\| \leq M$.

Then $T$ restricted to a ball with radius $R_{0}=M+\|p\|$ (as in the previous proof) has nonempty, closed, convex and weakly compact values. As a domain $Q$ for $T$ we put $\bar{U} \cap$ $B_{R_{0}}$. By repeating the proof from [40, Theorem 3.3] we are able to show that $T$ has weakly-weakly sequentially closed graph. Restricted to a weakly compact set $Q$ an operatot $T$ is sequentially weakly upper semi-continuous. This means, that the Kakutani fixed point theorem (Theorem 28) gives us a fixed point of $T$. The proof is complete.

\section{Pseudo-Solutions to Fractional Order Boundary Value Problem}

In this section, we are looking for sufficient conditions to ensure the existence of pseudo-solution to the boundary value problem (1) under the Pettis integrability assumption imposed on $f$. In order to obtain the existence of solutions of the problem (1), we can make use of Theorem 23.

Theorem 30. Let the assumptions of Theorem 23 be satisfied. Then the boundary value problem (1) has at least one pseudosolution $u \in C\left(I, E_{w}\right)$.

Proof. Firstly, we remark that, for any $v \in C[I, E]$, we have (according to Proposition 6) that $v(\cdot) \mathfrak{J}(\cdot) \in P[I, E]$ for $\mathfrak{\Im} \epsilon$ $L^{q}(I), q \in[1, \infty]$. Thus the integral boundary condition makes sense.

In account of Theorem 23 it can be easily seen that the integral equation (35) has a solution $v \in C[I, E]$. Let $v$ be a weak solution of (35). Then

$$
\begin{aligned}
& v(t)=\frac{t^{\alpha-\beta-1} l \Gamma(\alpha)}{\Gamma(\alpha-\beta)(1+\gamma)} \\
& +\int_{0}^{1}\left[G_{1}(t, s)+G_{2}(t, s)\right] \\
& \times f\left(s, I_{+}^{\beta} v(s), v(s)\right) d s \\
& =-\int_{0}^{t} \frac{(t-s)^{\alpha-\beta-1}}{\Gamma(\alpha-\beta)} f\left(s, I_{+}^{\beta} v(s), v(s)\right) d s+c t^{\alpha-\beta-1} \text {, } \\
& c=\frac{\Gamma(\alpha)}{\Gamma(\alpha-\beta)(1+\gamma)} \\
& \times\left[l+\int_{0}^{1} \frac{(1-s)^{\alpha-\beta-1} f\left(s, I_{+}^{\beta} v(s), v(s)\right)}{\Gamma(\alpha-\beta)} d s\right. \\
& \left.+\int_{0}^{1} h(s) f\left(s, I_{+}^{\beta} v(s), v(s)\right) d s\right] .
\end{aligned}
$$


By Lemma 14 and using $G_{1}(0, s)=G_{2}(0, s)=0$, a straightforward estimates show that

$$
v(0)=0, \quad I_{+}^{\beta} v(1)+\int_{0}^{1} \mathfrak{J}(\tau) I_{+}^{\beta} v(\tau) d \tau=l .
$$

Furthermore, we have

$$
v(t)=-I_{+}^{\alpha-\beta} f\left(t, I_{+}^{\beta} v(t), v(t)\right)+c t^{\alpha-\beta-1} .
$$

Thus for any $\varphi \in E^{*}$ we have

$$
\begin{aligned}
\varphi v(t) & =-\varphi\left(I_{+}^{\alpha-\beta} f\left(t, I_{+}^{\beta} v(t), v(t)\right)\right)+\varphi c t^{\alpha-\beta-1} \\
& =-I_{+}^{\alpha-\beta} \varphi\left(f\left(t, I_{+}^{\beta} v(t), v(t)\right)\right)+\varphi c t^{\alpha-\beta-1} .
\end{aligned}
$$

Operating by $I_{+}^{2-(\alpha-\beta)}$ on both sides of (59) and using the properties of fractional calculus in the space $L^{1}[0,1]$ (see, e.g., $[20,30])$ result in

$$
\begin{aligned}
I_{+}^{2-(\alpha-\beta)} \varphi v(t)= & -I_{+}^{2} \varphi\left(f\left(t, I_{+}^{\beta} v(t), v(t)\right)\right) \\
& +\varphi c \frac{\Gamma(\alpha-\beta)}{\Gamma(2)} t .
\end{aligned}
$$

Therefore

$$
\begin{aligned}
\varphi\left(I_{+}^{2-(\alpha-\beta)} v(t)\right)= & -I_{+}^{2} \varphi\left(f\left(t, I_{+}^{\beta} v(t), v(t)\right)\right) \\
& +\varphi c \frac{\Gamma(\alpha-\beta)}{\Gamma(2)} t .
\end{aligned}
$$

Thus

$$
\begin{aligned}
& \frac{d^{2}}{d t^{2}} \varphi\left(I_{+}^{2-(\alpha-\beta)} v(t)\right) \\
& \quad=-\varphi\left(f\left(t, I_{+}^{\beta} v(t), v(t)\right)\right) \text { a.e. on }[0,1] .
\end{aligned}
$$

That is, $v$ has the fractional pseudo-derivative of order $\alpha-$ $\beta \in(1,2)$ and satisfies

$$
D^{\alpha-\beta} v(t)=-f\left(t, I_{+}^{\beta} v(t), v(t)\right) \text { on }[0,1] .
$$

Therefore $v$ is a pseudo-solution to the problem (23). This together with Lemma 18 implies that the problem (1) has a pseudo-solution $u \in C\left(I, E_{w}\right)$ which completes the proof.

Now, we consider an example to illustrate our result.

Example 31. Let $\mathfrak{I} \in L^{\infty}(I)$ and assume that $E:=\ell^{2}(I)$ be the space of countably nonzero functions on $I:=[0,1]$ that are square-summable, under the $\ell^{2}$-norm. This is even a reflexive space. For each $t \in I$ we define $g(t):=e_{t} \in \ell^{2}(I)$ by

$$
e_{t}(s):= \begin{cases}1, & \text { if } s=t \\ 0, & \text { if } s \neq t .\end{cases}
$$

This function is Pettis, but not Bochner, integrable (it is not even strongly measurable $[22,41])$, and for any $\varphi \in \ell^{2}(I)$ we have $[22,41]$

$$
\varphi e_{t}=\sum_{\tau \in[0,1]} \varphi(\tau) e_{t}(\tau)= \begin{cases}0, & \text { if } \tau \neq t \\ \varphi(\tau), & \text { if } \tau=t\end{cases}
$$

Since $\left(\ell^{2}(I)\right)^{*}=\ell^{2}(I)$, the function $\varphi e_{t}$ is only countably nonzero for each $\varphi \in\left(\ell^{2}(I)\right)^{*}$. Hence $\varphi g=0$ a.e. (with respect to the Lebesgue measure).

Now, we investigate the existence of pseudo-solutions for the problem

$$
\begin{gathered}
D^{\alpha} u(t)+\mu D^{\beta} u(t)+\delta u(t)=g(t), \\
t \in[0,1], \quad \delta, \mu \in \mathbb{R}, \quad \alpha \in(1,2], \quad \beta \in(0,1), \quad \alpha>1+\beta, \\
u(1)+\int_{0}^{1} \mathfrak{J}(\tau) u(\tau) d \tau=l, \quad u(0)=0 .
\end{gathered}
$$

Let us define the function $f: I \times E \times E \rightarrow E$ by $f(t, x, y)=$ $\mu x+\delta y-g(t)$. Remark that for any $x, y \in C[I, E], f$ is Pettis, but not Bochner, integrable and satisfies, by suitable choice of $\delta, \mu$, all requirements of Theorem 30 with $\psi \equiv 1, \Omega(r)=$ $(\delta+\mu) r, p=1$, and $q=\infty$.

Therefore one cannot expect the existence of weak or strong solutions to the problem (66). In view of Theorem 30 the existence of pseudo-solutions is guaranteed. Since $f$ satisfies the requirements of Theorem 30 with $p=1, q=\infty$, the problem (66) has a pseudo-solution $u \in C\left(I, E_{w}\right)$ given by $u=I_{+}^{\beta} v$, where $v$ denotes the weak solution to the problem

$$
\begin{aligned}
v(t)= & \frac{l \Gamma(\alpha) t^{\alpha-\beta-1}}{\Gamma(\alpha-\beta)(1+\gamma)} \\
& +\int_{0}^{1} G(t, s)\left[\mu v(s)+\delta I_{+}^{\beta} v(s)-g(s)\right] d s, \\
& t \in(I) .
\end{aligned}
$$

That is, $v$ satisfies the problem

$$
\begin{aligned}
v(t)= & \frac{l \Gamma(\alpha) t^{\alpha-\beta-1}}{\Gamma(\alpha-\beta)(1+\gamma)} \\
& +\int_{0}^{1} G(t, s)\left[\mu v(s)+\delta I_{+}^{\beta} v(s)\right] d s \\
& -\int_{0}^{1} G(t, s) g(s) d s, \quad t \in(I) .
\end{aligned}
$$

Since $\int_{0}^{1} G(t, s) \varphi g(s) d s=0$, for each $\varphi \in \ell^{2}(I)$, we obtain $\int_{0}^{1} G(t, s) g(s) d s=0$. Hence

$$
\begin{aligned}
v(t) & =\frac{l \Gamma(\alpha) t^{\alpha-\beta-1}}{\Gamma(\alpha-\beta)(1+\gamma)}+\int_{0}^{1} G(t, s)\left[\mu \nu(s)+\delta I_{+}^{\beta} v(s)\right] d s \\
& =\frac{l \Gamma(\alpha) t^{\alpha-\beta-1}}{\Gamma(\alpha-\beta)(1+\gamma)}-I_{+}^{\alpha-\beta}\left[\mu \nu(s)+\delta I_{+}^{\beta} v(s)\right]+c t^{\alpha-\beta-1}
\end{aligned}
$$


where

$$
\begin{aligned}
c= & \frac{\Gamma(\alpha)}{\Gamma(\alpha-\beta)(1+\gamma)} \\
& \times\left[l+\int_{0}^{1} \frac{(1-s)^{\alpha-\beta-1}\left[\mu \nu(s)+\delta I_{+}^{\beta} v(s)\right]}{\Gamma(\alpha-\beta)} d s\right. \\
& \left.+\int_{0}^{1} h(s)\left[\mu v(s)+\delta I_{+}^{\beta} v(s)\right] d s\right] .
\end{aligned}
$$

Therefore

$$
v(t)+\mu I_{+}^{\alpha-\beta} v(t)+\delta I_{+}^{\alpha} v(t)=c^{*} t^{\alpha-\beta-1},
$$

where

$$
c^{*}=\left(c+\frac{l \Gamma(\alpha)}{\Gamma(\alpha-\beta)(1+\gamma)}\right) .
$$

Further

$$
I_{+}^{\beta} v(t)+\mu I_{+}^{\alpha-\beta} I_{+}^{\beta} v(t)+\delta I_{+}^{\alpha+\beta} v(t)=c^{*} \frac{\Gamma(\alpha-\beta)}{\Gamma(\alpha)} t^{\alpha-1} .
$$

Now if $u=I_{+}^{\beta} v$, we obtain

$$
u(t)+\mu I_{+}^{\alpha-\beta} u(t)+\delta I_{+}^{\alpha} u(t)=c^{*} \frac{\Gamma(\alpha-\beta)}{\Gamma(\alpha)} t^{\alpha-1} .
$$

Thus for every $\varphi \in\left(\ell^{2}(I)\right)^{*}$, we have

$$
\varphi u(t)+\mu I_{+}^{\alpha-\beta} \varphi u(t)+\delta I_{+}^{\alpha} \varphi u(t)=\varphi c^{*} \frac{\Gamma(\alpha-\beta)}{\Gamma(\alpha)} t^{\alpha-1} .
$$

Whence

$$
I_{+}^{2-\alpha} \varphi u(t)+\mu I_{+}^{2-\beta} \varphi u(t)+\delta I_{+}^{2} \varphi u(t)=\varphi c^{*} \frac{\Gamma(\alpha-\beta)}{\Gamma(2)} t .
$$

Moreover,

$$
\frac{d^{2}}{d t^{2}} \varphi\left(I_{+}^{2-\alpha} u(t)\right)+\frac{d^{2}}{d t^{2}} \varphi I_{+}^{2-\beta} u(t)+\delta \varphi u(t)=0 .
$$

Since

$$
\begin{aligned}
\frac{d^{2}}{d t^{2}} \varphi I_{+}^{2-\beta} u(t) & =\frac{d^{2}}{d t^{2}} I_{+}^{2-\beta} \varphi u(t) \\
& =\frac{d}{d t}\left(\frac{d}{d t} I_{+}^{1} I_{+}^{1-\beta} \varphi u(t)\right) \\
& =\frac{d}{d t} I_{+}^{1-\beta} \varphi u(t) \\
& =\frac{d}{d t} \varphi I_{+}^{1-\beta} u(t)
\end{aligned}
$$

we arrive, for every $\varphi \in E^{*}$, at $D^{\alpha} \varphi u(t)+\mu D^{\beta} \varphi u(t)+\delta \varphi u(t)=$ 0 for all $t \in[0,1]$ while $\varphi g=0$ a.e.

Finally

$$
D^{\alpha} \varphi u(t)+\mu D^{\beta} \varphi u(t)+\delta u(t)=\varphi e_{t}, \quad \text { a.e. }[0,1] \text {. }
$$

Then $u$ is a pseudo- (but not a weak or strong) solution to the problem (66).

\section{References}

[1] J. M. Gallardo, "Second-order differential operators with integral boundary conditions and generation of analytic semigroups," The Rocky Mountain Journal of Mathematics, vol. 30, no. 4, pp. 1265-1291, 2000.

[2] G. L. Karakostas and P. C. Tsamatos, "Multiple positive solutions of some Fredholm integral equations arisen from nonlocal boundary-value problems," Electronic Journal of Differential Equations, vol. 30, pp. 1-17, 2002.

[3] A. Lomtatidze and L. Malaguti, "On a nonlocal boundary value problem for second order nonlinear singular differential equations," Georgian Mathematical Journal, vol. 7, no. 1, pp. 133$154,2000$.

[4] M. Feng, D. Ji, and W. Ge, "Positive solutions for a class of boundary-value problem with integral boundary conditions in Banach spaces," Journal of Computational and Applied Mathematics, vol. 222, no. 2, pp. 351-363, 2008.

[5] L. Kong and Q. Kong, "Second-order boundary value problems with nonhomogeneous boundary conditions. II," Journal of Mathematical Analysis and Applications, vol. 330, no. 2, pp. 1393-1411, 2007.

[6] C. Pang, W. Dong, and Z. Wei, "Green's function and positive solutions of $n$th order $m$-point boundary value problem," Applied Mathematics and Computation, vol. 182, no. 2, pp. 12311239,2006

[7] S. H. Chen, J. Hu, L. Chen, and C. Wang, "Existence results for $n$-point boundary value problem of second order ordinary differential equations," Journal of Computational and Applied Mathematics, vol. 180, no. 2, pp. 425-432, 2005.

[8] Y. Guo, Y. Ji, and J. Zhang, “Three positive solutions for a nonlinear $n$ th-order $m$-point boundary value problem," Nonlinear Analysis: Theory, Methods \& Applications, vol. 68, no. 11, pp. 3485-3492, 2008.

[9] R. Ma and N. Castaneda, "Existence of solutions of nonlinear $m$-point boundary-value problems," Journal of Mathematical Analysis and Applications, vol. 256, no. 2, pp. 556-567, 2001.

[10] B. Yan, D. O’Regan, and R. P. Agarwal, "Nonexistence and existence of multiple positive solutions for superlinear three-point boundary value problems via index theory," Acta Mathematicae Applicatae Sinica (English Series), vol. 24, no. 4, pp. 529-540, 2008.

[11] Z. Bai and H. Lü, "Positive solutions for boundary value problem of nonlinear fractional differential equation," Journal of Mathematical Analysis and Applications, vol. 311, no. 2, pp. 495-505, 2005.

[12] W. C. Lian, F. H. Wong, and C. C. Yeh, "On the existence of positive solutions of nonlinear second order differential equations," Proceedings of the American Mathematical Society, vol. 124, no. 4, pp. 1117-1126, 1996. 
[13] A. M. Nahušev, "The Sturm-Liouville problem for a second order ordinary differential equation with fractional derivatives in the lower terms," Doklady Akademii Nauk SSSR, vol. 234, no. 2, pp. 308-311, 1977.

[14] D. Bugajewski and S. Szufla, "Kneser's theorem for weak solutions of the Darboux problem in Banach spaces," Nonlinear Analysis: Theory, Methods \& Applications, vol. 20, no. 2, pp. 169173, 1993.

[15] J. Chandra, V. Lakshmikantham, and A. R. Mitchell, "Existence of solutions of boundary value problems for nonlinear secondorder systems in a Banach space," Nonlinear Analysis, vol. 2, no. 2, pp. 157-168, 1978.

[16] B. Liu, "Positive solutions of a nonlinear four-point boundary value problems in Banach spaces," Journal of Mathematical Analysis and Applications, vol. 305, no. 1, pp. 253-276, 2005.

[17] D. O'Regan, "Weak solutions of ordinary differential equations in Banach spaces," Applied Mathematics Letters, vol. 12, no. 1, pp. 101-105, 1999.

[18] D. Ozdarska and S. Szufla, "Weak solutions of a boundary value problem for nonlinear ordinary differential equation of second order in Banach spaces," Mathematica Slovaca, vol. 43, no. 3, pp. 301-307, 1993.

[19] X. Zhang, M. Feng, and W. Ge, "Existence and nonexistence of positive solutions for a class of $n$ th-order three-point boundary value problems in Banach spaces," Nonlinear Analysis: Theory, Methods \& Applications, vol. 70, no. 2, pp. 584-597, 2009.

[20] H. A. H. Salem, "On the nonlinear Hammerstein integral equations in Banach spaces and application to the boundary value problem of fractional order," Mathematical and Computer Modelling, vol. 48, no. 7-8, pp. 1178-1190, 2008.

[21] H. A. H. Salem, "On the fractional order $m$-point boundary value problem in reflexive Banach spaces and weak topologies," Journal of Computational and Applied Mathematics, vol. 224, no. 2, pp. 565-572, 2009.

[22] M. Cichoń, "Weak solutions of differential equations in Banach spaces," Discussiones Mathematicae: Differential Inclusions, vol. 15, no. 1, pp. 5-14, 1995.

[23] M. Cichoń, "On solutions of differential equations in Banach spaces," Nonlinear Analysis: Theory, Methods \& Applications, vol. 60, no. 4, pp. 651-667, 2005.

[24] J. Diestel and J. J. Uhl, Jr., Vector Measures, vol. 15 of Mathematical Surveys, American Mathematical Society, Providence, RI, USA, 1977.

[25] B. J. Pettis, "On integration in vector spaces," Transactions of the American Mathematical Society, vol. 44, no. 2, pp. 277-304, 1938.

[26] E. Hille and R. S. Phillips, Functional Analysis and Semi-Groups, vol. 31 of American Mathematical Society Colloquium Publications, American Mathematical Society, Providence, RI, USA, 1957.

[27] O. Arino, S. Gautier, and J. P. Penot, "A fixed point theorem for sequentially continuous mappings with application to ordinary differential equations," Funkcialaj Ekvacioj, vol. 27, no. 3, pp. 273-279, 1984.

[28] N. Dinculeanu, "On Kolmogorov-Tamarkin and M. Riesz compactness criteria in function spaces over a locally compact group," Journal of Mathematical Analysis and Applications, vol. 89, no. 1, pp. 67-85, 1982.

[29] R. Gorenflo and S. Vessella, Abel Integral Equations, vol. 1461 of Lecture Notes in Mathematics, Springer, Berlin, Germany, 1991.

[30] S. G. Samko, A. A. Kilbas, and O. I. Marichev, Fractional Integrals and Derivatives, Gordon and Breach Science, Yverdon, Switzerland, 1993.
[31] K. Musiał, "Pettis integration of dominated functions," Atti del Seminario Matematico e Fisico dell'Università di Modena, vol. 38, no. 2, pp. 261-265, 1990.

[32] W. Lin, "Global existence theory and chaos control of fractional differential equations," Journal of Mathematical Analysis and Applications, vol. 332, no. 1, pp. 709-726, 2007.

[33] A. Szép, "Existence theorem for weak solutions of ordinary differential equations in reflexive Banach spaces," Studia Scientiarum Mathematicarum Hungarica, vol. 6, pp. 197-203, 1971.

[34] R. F. Geitz, "Pettis integration," Proceedings of the American Mathematical Society, vol. 82, no. 1, pp. 81-86, 1981.

[35] T. Maruyama, "A generalization of the weak convergence theorem in Sobolev spaces with application to differential inclusions in a Banach space," Proceedings of the Japan Academy A, vol. 77, no. 1, pp. 5-10, 2001.

[36] D. L. Azzam, C. Castaing, and L. Thibault, "Three boundary value problems for second order differential inclusions in Banach spaces," Control and Cybernetics, vol. 31, no. 3, pp. 659693, 2002.

[37] D. Azzam-Laouir and I. Boutana, "Application of Pettis integration to differential inclusions with three-point boundary conditions in Banach spaces," Electronic Journal of Differential Equations, vol. 173, pp. 1-8, 2007.

[38] B. Satco, "Second order three boundary value problem in Banach spaces via Henstock and Henstock-Kurzweil-Pettis integral," Journal of Mathematical Analysis and Applications, vol. 332, no. 2, pp. 919-933, 2007.

[39] K. El Amri and C. Hess, "On the Pettis integral of closed valued multifunctions," Set-Valued Analysis, vol. 8, no. 4, pp. 329-360, 2000.

[40] K. Cichoń and M. Cichoń, "Some applications of nonabsolute integrals in the theory of differential inclusions in Banach spaces," in Vector Measures, Integration and Related Topics, vol. 201 of Operator Theory: Advances and Applications, pp. 115-124, Birkhäuser, Basel, Switzerland, 2009.

[41] R. F. Geitz, "Geometry and the Pettis integral," Transactions of the American Mathematical Society, vol. 269, no. 2, pp. 535-548, 1982. 


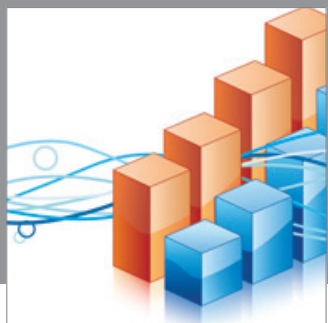

Advances in

Operations Research

mansans

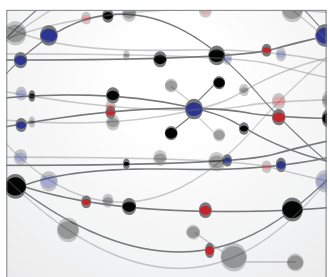

The Scientific World Journal
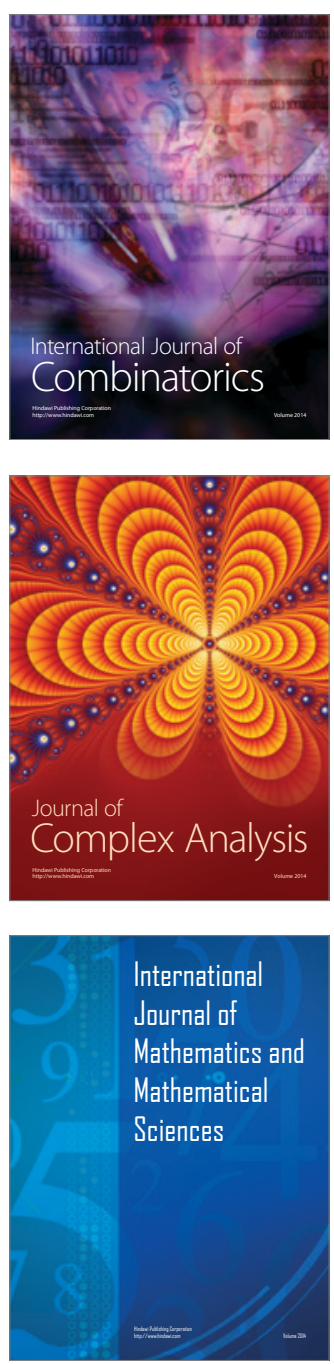
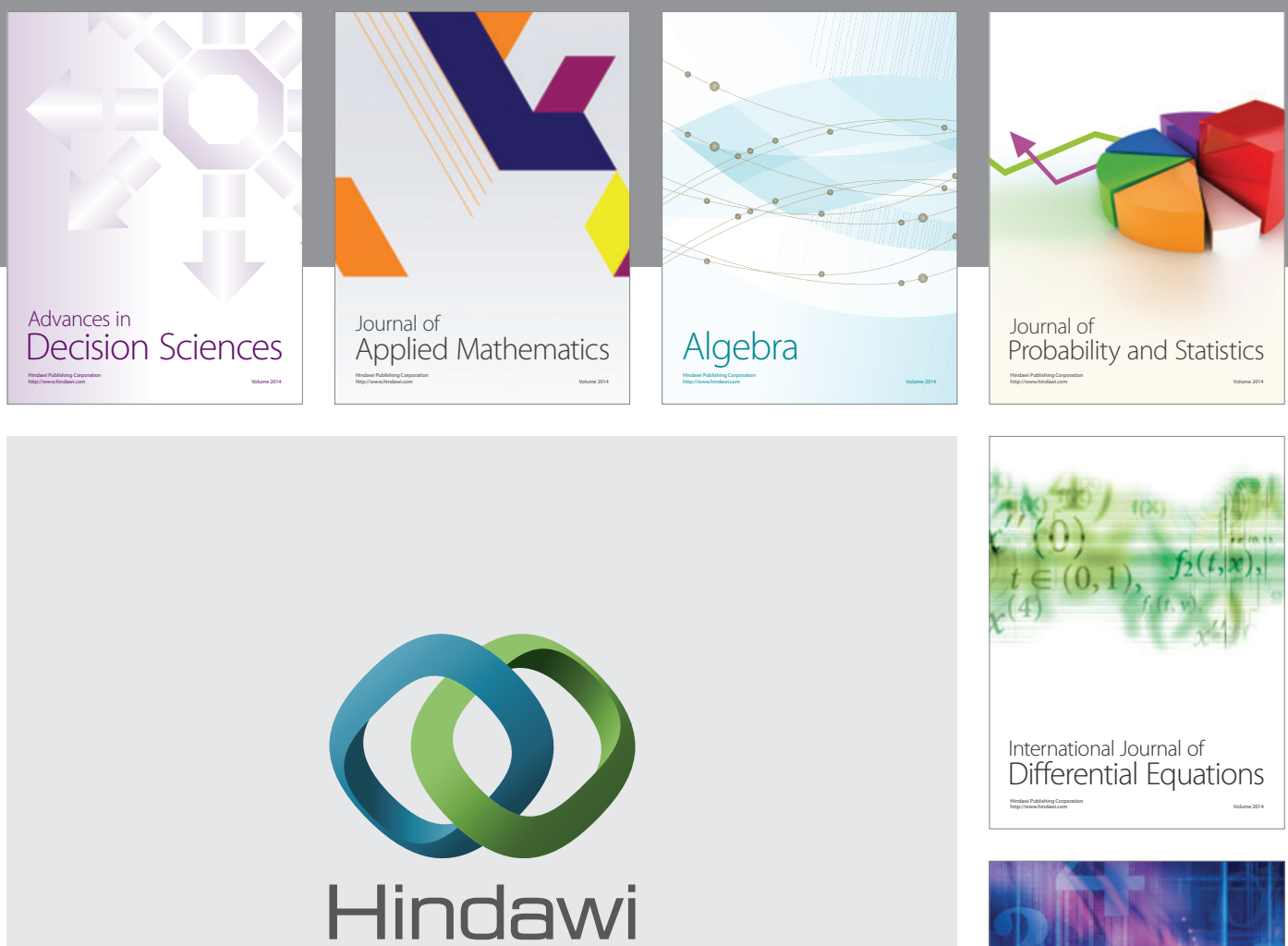

Submit your manuscripts at http://www.hindawi.com
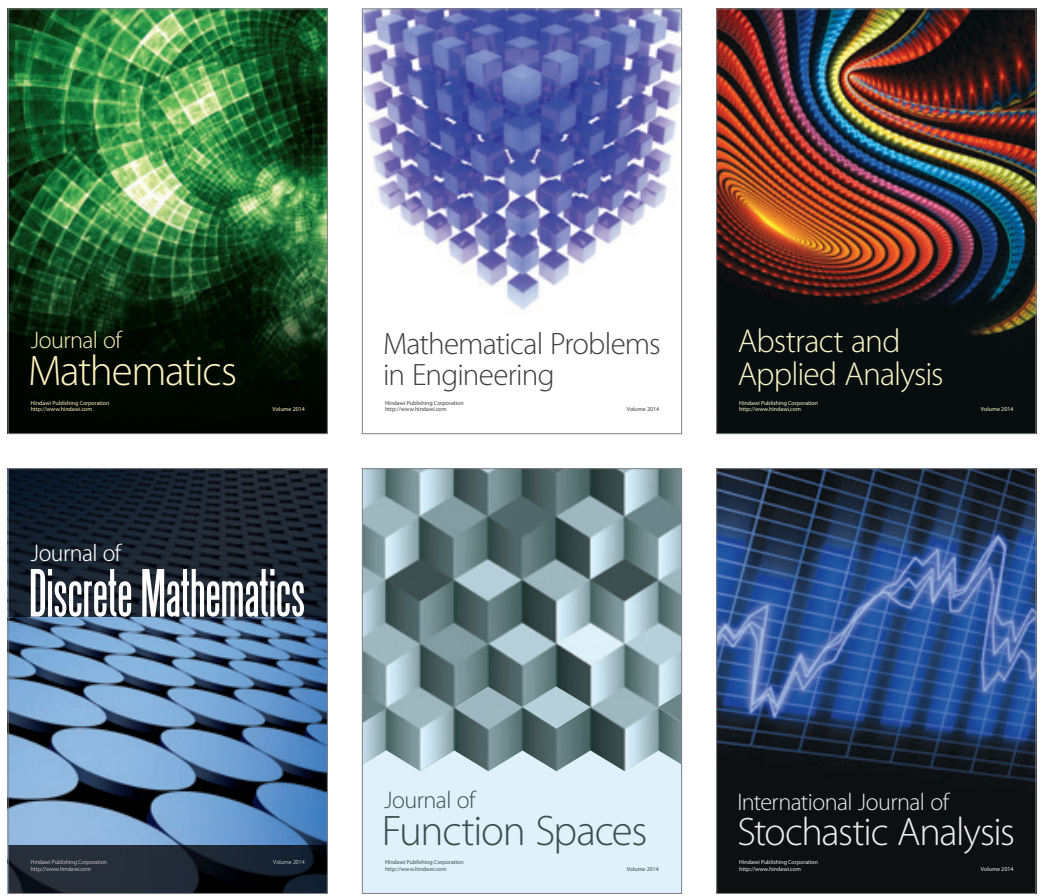

Journal of

Function Spaces

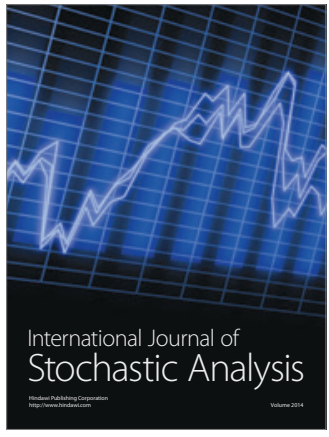

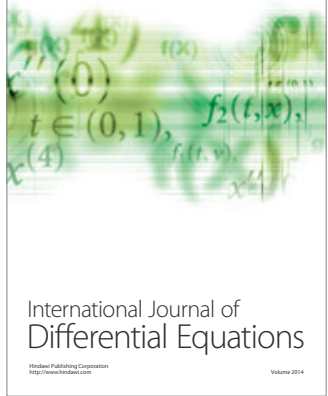
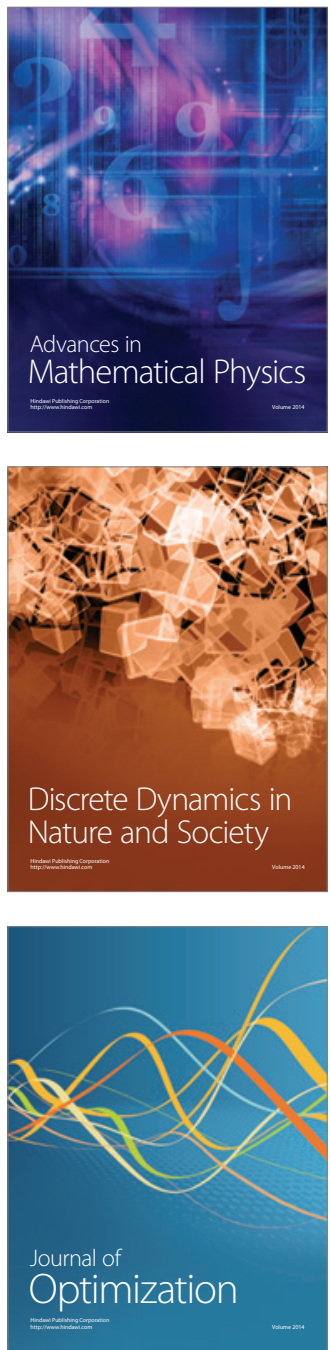